\title{
Role of Diabetes Mellitus in Liver Cirrhosis in the Anhui Region of China
}

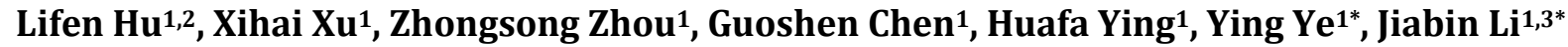 \\ ${ }^{1}$ Department of Infectious Diseases, the First Affiliated Hospital of Anhui Medical University, Hefei, China \\ ${ }^{2}$ Department of Center Laboratory, the First Hospital of Anhui Medical University, Hefei, China \\ ${ }^{3}$ Department of Infectious Diseases, the Affiliated Chaohu Hospital of Anhui Medical University, Chaohu, China \\ Email: "13856980361@139.com, "lijiabin948@vip.sohu.com
}

Received 30 October 2015; accepted 14 November 2015; published 20 November 2015

Copyright (C) 2015 by authors and OALib.

This work is licensed under the Creative Commons Attribution International License (CC BY).

http://creativecommons.org/licenses/by/4.0/

c) (i) Open Access

\begin{abstract}
Aims: The aim of this study was to investigate the frequency of diabetes mellitus (DM) among cirrhotic inpatients with different etiologies and the impact of DM on the prognosis of these patients. Methods: A retrospective study of the association between DM and cirrhosis was performed on 672 cirrhotic inpatients at the First Affiliated Hospital of Anhui Medical University from January 1, 2012 to March 1, 2013. Data were assessed using SPSS 20.0. Results: The DM prevalences involving different etiologies were $45.08 \%, 40.43 \%, 42.85 \%, 41.67 \%$, and $25.56 \%$ among 672 patients with alcoholic cirrhosis, cryptogenic cirrhosis, schistosomiasis, chronic hepatitis $\mathrm{C}$, and chronic hepatitis $B$, respectively. Multivariate analysis indicated that relative to non-diabetic patients, patients with DM were older (OR 2.83, 95\% CI $2.78-2.87, p<0.001$ ), had higher white blood cell levels (OR 3.01, 95\% CI $2.83-3.22, p=0.001$ ) and were more frequently Child-Pugh class C (OR 1.28, 95\% CI 1.14 - 1.55, $p<0.001)$; these differences were also statistically significant in univariate analysis. Univariate analysis suggested that the presence of DM was associated with a higher international normalized ratio $(p=0.013)$, a higher incidence of hepatic encephalopathy ( $p$ $=0.014)$, and a more frequent incidence of spontaneous bacterial peritonitis $(p<0.001)$. Conclusion: DM is extremely common among cirrhotic patients and is particularly prevalent among alcoholic cirrhosis patients in the examined region of China. The presence of DM in cirrhotic patients was strongly associated with an elevated Child-Pugh score and an accelerated progression of cirrhosis.
\end{abstract}

\section{Keywords}

Cirrhosis, Diabetes Mellitus, Child-Pugh Score, White Blood Cell, Encephalopathy, Spontaneous Bacterial Peritonitis

Subject Areas: Internal Medicine

${ }^{*}$ Corresponding authors.

How to cite this paper: Hu, L.F., Xu, X.H., Zhou, Z.S., Chen, G.S., Ying, H.F., Ye, Y. and Li, J.B. (2015) Role of Diabetes Mellitus in Liver Cirrhosis in the Anhui Region of China. Open Access Library Journal, 2: e2101.

http://dx.doi.org/10.4236/oalib.1102101 


\section{Introduction}

Liver cirrhosis which ultimately results in liver failure and portal hypertension is a common chronic liver disease and a major cause of death throughout the world, particularly in China. Because the liver plays an important role in carbohydrate metabolism and controls blood glucose levels through glycogenogenesis and glycogenolysis [1] [2], patients with cirrhosis may have alterations in glucose homeostasis; these patients often exhibit impaired glucose tolerance (IGT) or eventually develop diabetes mellitus (DM) [3]. In addition, increasing numbers of cirrhotic patients are complicated with DM; at present, DM is a common comorbidity among patients with liver cirrhosis. In cirrhotic patients, DM is reportedly associated with an increased risk for the continued development of cirrhosis [4]. Both liver cirrhosis and DM represent difficult challenges for clinicians and patients with respect to therapeutic and prognostic implications, particularly in China, which has large numbers of patients with both cirrhosis and DM. Several studies have reported a prevalence of DM among cirrhosis patients of $20 \%-40 \%$ [3] [5]-[7], and this prevalence is reportedly higher among patients with hepatitis C virus (HCV)-related cirrhosis than among patients with cirrhosis caused by other factors [8]-[11].

However, little information is available regarding the impact of cirrhosis with different etiologies on the occurrence of DM among Chinese patients and the relationship between DM and complications of decompensated cirrhosis. The aims of this study were to explore the relationship between different etiologies of cirrhosis and the incidence of IGT or DM and to evaluate whether DM can accelerate the progression of liver disease among cirrhotic patients.

\section{Materials and Methods}

\subsection{Patients and Methods}

From January 1, 2012 to March 1, 2013, 672 patients with liver cirrhosis with a single etiology who visited the Infection Department and the Digestive System Department of the First Affiliated Hospital of Anhui Medical University were involved in this study. The study was conducted in accordance with the guidelines of the Declaration of Helsinki and Good Clinical Practice and was approved locally by the Ethics Committee of the First Affiliated Hospital of Anhui Medical University. Liver function tests were performed to get the values of total bilirubin, alanine aminotransferases (ALT), aspartate aminotransferase (AST), serum albumin, prealbumin (PA) and the international normalized ratio (INR) in patients, other Laboratory tests including viral markers (hepatitis B surface antigen and anti-HCV) by Polymerase chain reaction PCR, white blood cells (WBC) by routine blood test, and fasting blood glucose by glucose oxidase method were performed; in addition, an oral glucose tolerance test (OGTT) was performed as needed. Autoimmune markers, liver ultrasonography images and liver CT scans were also examined. Detailed clinical characteristics were recorded, including each patient's sex; age; body mass index (BMI); alcohol consumption; etiology of cirrhosis; and history of ascites, hepatic encephalopathy (HE) which were diagnosed according to West Haven criteria [12], spontaneous bacterial peritonitis (SBP) which were diagnosed by EASL clinical practice guidelines [13] and DM. The severity of liver cirrhosis was classified using Child-Pugh scores; in particular, cases with Child-Pugh scores of 5 - 6, 7 - 9 and 10 - 15 were categorized as class A, class B, and class $\mathrm{C}$, respectively.

Diagnoses of cirrhosis were established using a combination of clinical features, laboratory tests, radiological and histological examinations, and liver biopsy findings (if available). The presence of DM was established based on a history of DM, the use of an oral hypoglycemic medication or insulin, fasting blood glucose levels > $126 \mathrm{mg} / \mathrm{dL}$ for two consecutive days, a blood glucose level $>200 \mathrm{mg} / \mathrm{dL} 2 \mathrm{~h}$ after an OGTT, or a random blood glucose level $>200 \mathrm{mg} / \mathrm{dL}$. Patients were diagnosed with IGT if they exhibited a fasting glucose concentration $\geq$ $110 \mathrm{mg} / \mathrm{dL}$ and $<126 \mathrm{mg} / \mathrm{dL}$ or a glucose concentration $\geq 140 \mathrm{mg} / \mathrm{dL}$ and $<200 \mathrm{mg} / \mathrm{dL}$ at $2 \mathrm{~h}$ after an OGTT.

\subsection{Statistical Analysis}

Data were analyzed using SPSS, version 20.0 (SPSS Inc., USA). Quantitative variables were expressed as means \pm standard deviation (SD) or as medians (range). Univariate analysis was performed by utilizing the independent Student's $t$-test or the Mann-Whitney test for between-group comparisons of continuous variables and chi-squared tests for between-group comparisons of qualitative data. Multiple logistic regression analysis was also used for between-group comparisons of differences in clinical and biochemical variables. A two-sided 
$p$-value $<0.05$ was considered to be statistically significant.

\section{Results}

\subsection{Prevalence of IGT and DM in Cirrhotic Patients}

Among the 672 cirrhotic patients, 88 patients (13.1\%) were diagnosed with IGT, and 210 patients (31.25\%) were diagnosed with DM; thus, in total, 298 patients (44.35\%) were diagnosed with IGT or DM. The prevelance of DM and IGT in cirrhotic patients with different etiologies were indicated in Table 1.

\subsection{Clinical Characteristics of Patients with and without IGT or DM}

Comparison of clinical and biochemical characteristics between cirrhotic patients with IGT or DM and without among 672 patients by univariate analysis are presented in Table 2 and Table 3 . The results of $t$-tests indicated that the DM group was significantly older than the patients without IGT or DM ( $55.95 \pm 12.36$ vs. $51.09 \pm 12.36$ years, $p<0.001)$ and the IGT group was also older than the patients without IGT or DM $(52.88 \pm 12.74$ vs. $51.09 \pm 12.36$ years, $p=0.226$ ). In comparisons of patients with DM and patients without DM or IGT, patients with DM not only had significantly higher WBC levels $(5.76 \pm 2.96$ vs. $4.46 \pm 3, p<0.001)$ and INR levels $(1.65 \pm 0.62$ vs. $1.50 \pm 0.66, p=0.013)$ as well as significantly higher incidences of HE $(17.14 \%$ vs. $10.43 \%, p$ $=0.014)$ and SBP $(25.23 \%$ vs. $15.24 \%, p<0.001)$ but also were more frequently Child-Pugh class C (28.57\% vs. $16.58 \%, p<0.001)$. These comparisons revealed no associations between the presence of DM and sex, BMI, total bilirubin, ALT, AST, albumin, INR, or PA. Comparisons of patients with IGT and patients without DM or IGT revealed that patients with IGT had significantly higher WBC levels (5.68 \pm 1.98 vs. $4.46 \pm 3, p<0.001$ ) and were more frequently Child-Pugh class C (32.95\% vs. $16.58 \%, p<0.001)$.

As indicated in Table 2 and Table 3, multiple logistic regression analysis that compared patients with DM and patients without IGT or DM indicated that older age (OR 2.83, 95\% CI $2.78-2.87, p<0.001$ ), elevated WBC levels (OR 3.01, 95\% CI $2.83-3.22, p=0.001$ ) and a greater frequency of Child-Pugh class C (OR 1.28, $95 \%$ CI $1.14-1.55, p<0.001$ ) remained significantly associated with DM; in contrast, the presence of DM was no longer significantly associated with INR level or the incidence of HE or SBP. In multiple logistic regression analysis, WBC levels (OR 3, 95\% CI $2.79-3.26, p=0.008)$ and the frequency of Child-Pugh class C (OR 1.31, $95 \%$ CI $1.13-1.78, p=0.008$ ) remained significantly associated with IGT.

\section{Discussion}

It has long been known that cirrhosis, glucose abnormalities, and DM are associated; several contributing factors to these associations, such as insulin resistance, hyperinsulinemia, and reduced glucose uptake by cirrhotic livers, have been identified [14] [15]. In addition, Perry recently reported that white adipose tissue-derived hepatic acetyl CoA is linked to inflammation-induced hepatic insulin resistance, which is associated with obesity and type 2 diabetes [16].

Various etiologies, such as alcoholic hepatitis, CHC, CHB and nonalcoholic fatty liver disease, are common causes of cirrhosis, and the different etiologies of liver cirrhosis may cause differences in the incidence rates of DM among patients with cirrhosis. Several studies have reported a high prevalence of DM among patients infected with HCV or HBV [3] [11] [17], and observations have indicated that diabetes occurs more frequently among HCV-infected patients than among HBV-infected patients. The results of this study also revealed that the

Table 1. Prevalences of diabetes mellitus and impaired glucose tolerance among cirrhotic patients with different etiologies.

\begin{tabular}{ccccccc}
\hline Group & CHB & ALD* & CHC & $\begin{array}{c}\text { Autoimmune } \\
\text { Diseases }\end{array}$ & $\begin{array}{c}\text { Schistosomiasis } \\
\text { Cirrhosis }^{\dagger}\end{array}$ \\
\hline N & 403 & 122 & 24 & 62 & 47 & 14 \\
DM, N (\%) & $103(25.56)$ & $55(45.08)$ & $10(41.67)$ & $19(30.65)$ & $6(42.85)$ & $19(40.43)$ \\
IGT or DM, N (\%) & $155(38.46)$ & $75(61.48)$ & $10(41.67)$ & $26(41.94)$ & $6(42.85)$ & $27(57.45)$ \\
\hline
\end{tabular}

DM: diabetes mellitus, IGT: impaired glucose tolerance, CHB: chronic hepatitis B virus, ALD: alcoholic liver disease, CHC: chronic hepatitis C virus. DM incidence comparison: ${ }^{*} p<0.001$ in ALD vs. CHB, $p=0.059$ in ALD vs. autoimmune-disease group, ${ }^{\dagger} p=0.03$ in CHB vs. the cryptogenic group. IGT or DM incidence comparison: ${ }^{*} p<0.001$ in ALD vs. CHB, $p=0.012$ in ALD vs. autoimmune-disease group, ${ }^{\dagger} p=0.012$ in $\mathrm{CHB}$ vs. the cryptogenic group. 
Table 2. Comparison of clinical and biochemical variables among cirrhotic patients with DM and cirrhotic patients without IGT or DM.

\begin{tabular}{|c|c|c|c|c|c|c|c|}
\hline \multirow{2}{*}{\multicolumn{2}{|c|}{ Index }} & \multirow{2}{*}{ Without DM or IGT } & \multirow{2}{*}{ DM } & \multirow{2}{*}{$\begin{array}{c}\text { Univariate Analysis } \\
\text { p }\end{array}$} & \multicolumn{3}{|c|}{ Multivariate Analysis } \\
\hline & & & & & OR & $95 \% \mathrm{CI}$ & $\mathrm{p}$ \\
\hline \multicolumn{2}{|l|}{$\mathrm{N}$} & 374 & 210 & & & & \\
\hline \multicolumn{2}{|l|}{ Age (years) } & $51.09 \pm 12.36$ & $55.95 \pm 12.36$ & 0.000 & 2.83 & $2.78-2.87$ & 0.000 \\
\hline \multicolumn{2}{|l|}{ Sex: male, N (\%) } & $241(64.44)$ & $142(67.62)$ & 0.401 & 2.1 & $1.63-3.05$ & 0.149 \\
\hline \multicolumn{2}{|l|}{$\operatorname{BMI}\left(\mathrm{kg} / \mathrm{m}^{2}\right)$} & $22.92 \pm 2.83$ & $22.98 \pm 3.02$ & 0.881 & 2.71 & $2.55-2.89$ & 0.906 \\
\hline \multicolumn{2}{|c|}{ Total bilirubin (umol/l) } & $31.9(4.2-423)$ & $28.4(7.7-461)$ & 0.755 & 2.71 & $2.70-2.72$ & 0.110 \\
\hline \multicolumn{2}{|l|}{ ALT (units/l) } & $48(15$ - 1516) & $46(18$ - 1744) & 0.389 & 2.72 & $2.72-2.73$ & 0.164 \\
\hline \multicolumn{2}{|l|}{ AST (units/l) } & $68(28$ - 1495) & $57(19$ - 1586) & 0.309 & 2.71 & $2.71-2.74$ & 0.127 \\
\hline \multicolumn{2}{|l|}{ WBC $\left(* 10^{\wedge} 9\right)$} & $4.46 \pm 3$ & $5.76 \pm 2.96$ & 0.000 & 3.01 & $2.83-3.22$ & 0.001 \\
\hline \multicolumn{2}{|l|}{ Albumin (g/l) } & $31.25 \pm 7.51$ & $30.35 \pm 7.12$ & 0.085 & 2.72 & $2.66-2.73$ & 0.928 \\
\hline \multicolumn{2}{|l|}{ PA (mg/l) } & $85(10-335)$ & $94(12-336)$ & 0.134 & 2.72 & $2.71-2.73$ & 0.589 \\
\hline \multicolumn{2}{|l|}{ INR } & $1.50 \pm 0.66$ & $1.65 \pm 0.62$ & 0.013 & 3.36 & $2.43-5.23$ & 0.224 \\
\hline \multicolumn{2}{|l|}{ HE, N (\%) } & 39 (10.43) & 36 (17.14) & 0.014 & 3.44 & $2.07-8.96$ & 0.407 \\
\hline \multicolumn{2}{|l|}{ SBP, N (\%) } & $57(15.24)$ & 59 (25.23) & 0.000 & 3.71 & $2.25-8.37$ & 0.270 \\
\hline \multirow{2}{*}{ Child-Pugh score } & B, N (\%) & $98(26.2)$ & $63(30.00)$ & 0.325 & 1.68 & $1.36-2.42$ & 0.016 \\
\hline & C, N (\%) & 62 (16.58) & 60 (28.57) & 0.000 & 1.28 & $1.14-1.55$ & 0.000 \\
\hline
\end{tabular}

Results are expressed as mean \pm standard deviation (SD), frequencies (\%) or medians (range). ALT: alanine aminotransferases, AST: aspartate aminotransferase, BMI: body mass index, DM: diabetes mellitus, IGT: impaired glucose tolerance, PA: prealbumin, INR: international normalized ratio, HE: hepatic encephalopathy, SBP: spontaneous bacterial peritonitis.

Table 3. Comparison of clinical and biochemical variables among cirrhotic patients with IGT and cirrhotic patients without IGT or DM.

\begin{tabular}{|c|c|c|c|c|c|c|c|}
\hline \multirow{2}{*}{\multicolumn{2}{|c|}{ Index }} & \multirow{2}{*}{ Without DM or IGT } & \multirow{2}{*}{ IGT } & \multirow{2}{*}{$\begin{array}{c}\text { Univariate Analysis } \\
\mathrm{p}\end{array}$} & \multicolumn{3}{|c|}{ Multivariate Analysis } \\
\hline & & & & & OR & $95 \% \mathrm{CI}$ & $\mathrm{p}$ \\
\hline \multicolumn{2}{|l|}{$\mathrm{N}$} & 374 & 88 & & & & \\
\hline \multicolumn{2}{|l|}{ Age (years) } & $51.09 \pm 12.36$ & $52.88 \pm 12.74$ & 0.226 & 2.76 & $2.71-2.82$ & 0.116 \\
\hline \multicolumn{2}{|l|}{ Sex: male, N (\%) } & $241(64.44)$ & $142(67.62)$ & 0.266 & 2.18 & $1.57-3.85$ & 0.371 \\
\hline \multicolumn{2}{|l|}{$\operatorname{BMI}\left(\mathrm{kg} / \mathrm{m}^{2}\right)$} & $22.92 \pm 2.83$ & $22.38 \pm 3.37$ & 0.124 & 2.54 & $2.35-2.77$ & 0.118 \\
\hline \multicolumn{2}{|c|}{ Total bilirubin (umol/l) } & $31.9(4.2-423)$ & $40(5.1$ - 434) & 0.369 & 2.71 & $2.71-2.72$ & 0.252 \\
\hline \multicolumn{2}{|l|}{ ALT (units/l) } & $48(15-1516)$ & $46(19-1610)$ & 0.6 & 2.72 & $2.72-2.73$ & 0.372 \\
\hline \multicolumn{2}{|l|}{ AST (units/l) } & $68(28-1495)$ & $67(23-1423)$ & 0.664 & 2.72 & $2.71-2.72$ & 0.461 \\
\hline \multicolumn{2}{|l|}{ WBC $\left(* 10^{\wedge} 9\right)$} & $4.46 \pm 3$ & $5.68 \pm 1.98$ & 0.004 & 3 & $2.79-3.26$ & 0.008 \\
\hline \multicolumn{2}{|l|}{ Albumin (g/l) } & $31.25 \pm 7.51$ & $30.72 \pm 8.26$ & 0.349 & 2.72 & $2.66-2.79$ & 0.905 \\
\hline \multicolumn{2}{|l|}{ PA (mg/l) } & $85(10-335)$ & $86.5(13-367)$ & 0.118 & 2.72 & $2.71-2.73$ & 0.868 \\
\hline \multicolumn{2}{|l|}{ INR } & $1.50 \pm 0.66$ & $1.56 \pm 0.52$ & 0.386 & 2.33 & $1.68-3.95$ & 0.498 \\
\hline \multicolumn{2}{|l|}{ HE, N (\%) } & 39 (10.43) & $12(13.64)$ & 0.389 & 2.82 & $1.63-9.10$ & 0.924 \\
\hline \multicolumn{2}{|l|}{ SBP, N (\%) } & $57(15.24)$ & $21(23.86)$ & 0.054 & 2.85 & $1.70-7.81$ & 0.896 \\
\hline \multirow{2}{*}{ Child-Pugh score } & B, N (\%) & $98(26.2)$ & $25(28.41)$ & 0.674 & 1.72 & $1.31-2.92$ & 0.078 \\
\hline & C, N (\%) & 62 (16.58) & 29 (32.95) & 0.002 & 1.31 & $1.13-1.78$ & 0.001 \\
\hline
\end{tabular}

Results are expressed as mean \pm standard deviation (SD), frequencies (\%) or medians (range). ALT: alanine aminotransferases, AST: aspartate aminotransferase, BMI: body mass index, DM: diabetes mellitus, IGT: impaired glucose tolerance, PA: prealbumin, INR: international normalized ratio, HE: hepatic encephalopathy, SBP: spontaneous bacterial peritonitis. 
incidence of DM was higher among patients with HCV-induced cirrhosis than among patients with HBV-induced cirrhosis. One concerning finding was the presence of a strong association between alcohol-related cirrhosis and DM. Among the examined etiologies of cirrhosis, alcohol-related cirrhosis was associated with the highest incidence of DM or IGT; this incidence was also higher than the corresponding incidences reported in other studies of alcohol-related cirrhosis. Excessive alcohol intake can lead to obesity, which is linked to inflammation-induced hepatic insulin resistance and to decreased insulin-mediated glucose uptake under acute conditions. Chronic alcoholism may damage pancreatic islet cells, which may lead to the development of DM or IGT.

For cirrhotic patients, the coexistence of DM with cirrhosis might increase the risk of adverse progression and cause severe complications. According to prior studies, increases in orocecal transit time and intestinal bacterial growth due to diabetes may increase blood ammonia levels, which could lead to a high prevalence of HE among cirrhotic patients with diabetes [18] [19]. However, in this study, although univariate analysis indicated that diabetes was significantly associated with the incidence of $\mathrm{HE}$, the association between DM and the presence of HE did not reach statistical significance in multiple logistic regression analysis after adjusting for several other factors, such as age, BMI, WBC, INR, SBP, total bilirubin, ALT, AST, albumin, and PA. SBP is another important complication among cirrhotic patients that requires hospitalization and treatment with antimicrobial agents; this condition can lead to poor quality of life and shortened survival times [18] [20] [21]. A prior study reported that among patients with cirrhosis, the presence of DM in patients was associated with an increased risk of SBP [21]. In the present study, univariate analysis indicated that the prevalence of SBP was significantly higher among patients with DM than among patients without DM $(25.23 \%$ vs. $15.43 \%, p<0.001)$; however, this difference was not statistically significant in multivariate analysis after adjusting for several other factors.

In the present study, in both univariate and multivariate analysis, older age, higher WBC levels and elevated Child-Pugh scores were strongly and significantly associated with DM in cirrhotic patients. Higher WBC levels may imply an increased susceptibility to (bacterial) infections among cirrhotic patients with DM relative to cirrhotic patients without DM; this suggestion is consistent with previous observations that DM is associated with a greater incidence of not only SBP but also respiratory and urinary tract infections in patients with cirrhosis [22].

The Child-Pugh score is important for assessing the prognosis of cirrhosis. The percentage of patients who were Child-Pugh class $\mathrm{C}$ was significantly higher in both the DM group and the IGT group relative to non-diabetic cirrhosis patients; this finding may indicate that diabetes appears to result in the accelerating deterioration of liver disease and more severe liver function abnormalities in cases of decompensation cirrhosis. The data of this study revealed that in cirrhotic patients, diabetes does not significantly affect various biochemical parameters, including BMI, total bilirubin, ALT, AST, albumin, and PA levels. These results are similar to the findings of previous studies [23].

\section{Conclusion}

In conclusion, DM is extremely common among patients with liver cirrhosis. One noteworthy finding is that in the examined Chinese region, DM was particularly prevalent among patients with alcoholic cirrhosis. In addition, for cirrhotic patients, the presence of DM increased the risk of elevated Child-Pugh scores and accelerated the progression of cirrhosis. Therefore, clinicians should devote a great deal of attention to the incidence of diabetes in liver cirrhosis, particularly in cases involving alcoholic cirrhosis.

\section{Acknowledgments}

This study was supported by the Natural Science Foundation of China (No. 81172737 and No. 81101313).

\section{References}

[1] Barthel, A. and Schmoll, D. (2003) Novel Concepts in Insulin Regulation of Hepatic Gluconeogenesis. American Journal of Physiology - Endocrinology and Metabolism, 285, 685-692. http://dx.doi.org/10.1152/ajpendo.00253.2003

[2] Picardi, A., D’Avola, D., Gentilucci, U.V., et al. (2006) Diabetes in Chronic Liver Disease: From Old Concepts to New Evidence. Diabetes/Metabolism Research and Reviews, 22, 274-283. http://dx.doi.org/10.1002/dmrr.636

[3] Hickman, I.J. and Macdonald, G.A. (2007) Impact of Diabetes on the Severity of Liver Disease. The American Journal of Medicine, 120, 829-834. http://dx.doi.org/10.1016/j.amjmed.2007.03.025 
[4] Quintana, J.O., Garcia-Compean, D., Gonzdlez, J.A., Pérez, J.Z., González, F.J., et al. (201l) The Impact of Diabetes Mellitus in Mortality of Patients with Compensated Liver Cirrhosis a Prospective Study. Annals of Hepatology, 10, 56-62.

[5] Zein, N.N., Abdulkarim, A.S., Wiesner, R.H., Egan, K.S. and Persing, D.H. (2000) Prevalence of Diabetes Mellitus in Patients with End-Stage Liver Cirrhosis Due to Hepatitis C, Alcohol, or Cholestatic Disease. Journal of Hepatology, 32, 209-217. http://dx.doi.org/10.1016/S0168-8278(00)80065-3

[6] Holstein, A., Hinze, S., Thiessen, E., Plaschke, A. and Egberts, E.H. (2002) Clinical Implications of Hepatogenous Diabetes in Liver Cirrhosis. Journal of Gastroenterology and Hepatology, 17, 677-681. http://dx.doi.org/10.1046/j.1440-1746.2002.02755.x

[7] Wlazlo, N., Beijers, H.J., Schoon, E.J., Sauerwein, H.P. and Stehouwer, C.D. and Bravenboer, B. (2010) High Prevalence of Diabetes Mellitus in Patients with Liver Cirrhosis. Diabetic Medicine, 27, 1308-1311. http://dx.doi.org/10.1111/j.1464-5491.2010.03093.x

[8] Huang, J.F., Yu, M.L., Dai, C.Y. and Chuang, W.L. (2013) Glucose Abnormalities in Hepatitis C Virus Infection. Kaohsiung Journal of Medical Sciences, 29, 61-68. http://dx.doi.org/10.1016/j.kjms.2012.11.001

[9] Sporea, I., Sirli, R., Hogea, C., Sink, A.A. and Serban, V. (2009) Diabetes Mellitus and Chronic HCV Infection. Romanian Journal of Internal Medicine, 47, 141-147.

[10] Lonardo, A., Adinolfi, L.E., Petta, S., Craxì, A. and Loria, P. (2009) Hepatitis C and Diabetes: The Inevitable Coincidence? Expert Review of Anti-Infective Therapy, 7, 293-308. http://dx.doi.org/10.1586/eri.09.3

[11] Elgouhari, H.M., Zein, C.O., Hanouneh, I., Feldstein, A.E. and Zein, N.N. (2009) Diabetes Mellitus Is Associated with Impaired Response to Antiviral Therapy in Chronic Hepatitis C Infection. Digestive Diseases and Sciences, 54, 26992705. http://dx.doi.org/10.1007/s10620-008-0683-2

[12] Ferenci, P., Lockwood, A., Mullen, K., Tarter, R., Weissenborn, K. and Blei, A.T. (2002) Hepatic Encephalopathy: Definition, Nomenclature, Diagnosis, and Quantification: Final Report of the Working Party at the 11th World Congresses of Gastroenterology, Vienna, 1998. Hepatology, 35, 716-721. http://dx.doi.org/10.1053/jhep.2002.31250

[13] Garcia-Compean, D., Jaquez-Quintana, J.O., Gonzalez-Gonzalez, J.A. and Maldonado-Garza, H. (2009) Liver Cirrhosis and Diabetes: Risk Factors, Pathophysiology, Clinical Implications and Management. World Journal of Gastroenterology, 15, 280-288. http://dx.doi.org/10.3748/wjg.15.280

[14] European Association for the Study of the L (2010) EASL Clinical Practice Guidelines on the Management of Ascites, Spontaneous Bacterial Peritonitis, and Hepatorenal Syndrome in Cirrhosis. Journal of Hepatology, 53, 397-417. http://dx.doi.org/10.1016/j.jhep.2010.05.004

[15] Nielsen, M.F., Caumo, A., Aagaard, N.K., Chandramouli, V., Schumann, W.C., Landau, B.R., et al. (2005) Contribution of Defects in Glucose Uptake to Carbohydrate Intolerance in Liver Cirrhosis: Assessment during Physiological Glucose and Insulin Concentrations. American Journal of Physiology—Gastrointestinal and Liver Physiology, 288, 1135-1143. http://dx.doi.org/10.1152/ajpgi.00278.2004

[16] Perry, R.J., Camporez, J.P.G., Kursawe, R., et al. (2015) Hepatic Acetyl CoA Links Adipose Tissue Inflammation to Hepatic Insulin Resistance and Type 2 Diabetes. Cell, 160, 1-14. http://dx.doi.org/10.1016/j.cell.2015.01.012

[17] Papatheodoridis, G.V., Chrysanthos, N., Savvas, S., et al. (2006) Diabetes Mellitus in Chronic Hepatitis B and C: Prevalence and Potential Association with the Extent of Liver Fibrosis. Journal of Viral Hepatitis, 13, 303-310. http://dx.doi.org/10.1111/j.1365-2893.2005.00677.x

[18] Ampuero, J., Ranchal, I., Del Mar Díaz-Herrero, M., Del Campo, J.A., Bautista, J.D. and Romero-Gómez, M. (2013) Role of Diabetes Mellitus on Hepatic Encephalopathy. Metabolic Brain Disease, 28, 277-279. http://dx.doi.org/10.1007/s11011-012-9354-2

[19] Butt, Z., Jadoon, N.A., Salaria, O.N., Mushtaq, K., Riaz, I.B., Shahzad, A., et al. (2013) Diabetes Mellitus and Decompensated Cirrhosis: Risk of Hepatic Encephalopathy in Different Age Groups. Journal of Diabetes, 5, 449-455. http://dx.doi.org/10.1111/1753-0407.12067

[20] Poh, Z. and Chang, P.E. (2012) A Current Review of the Diagnostic and Treatment Strategies of Hepatic Encephalopathy. International Journal of Hepatology, 2012, Article ID: 480309. http://dx.doi.org/10.1155/2012/480309

[21] Wlazlo, N., van Greevenbroek, M.M., Curvers, J., Schoon, E.J., Friederich, P., Twisk, J.W., et al. (2013) Diabetes Mellitus at the Time of Diagnosis of Cirrhosis Is Associated with Higher Incidence of Spontaneous Bacterial Peritonitis, but Not with Increased Mortality. Clinical Science, 125, 341-348. http://dx.doi.org/10.1042/CS20120596

[22] Diaz, J., Monge, E., Roman, R. and Ulloa, V. (2008) Diabetes as a Risk Factor for Infections in Cirrhosis. American Journal of Gastroenterology, 103, 248. http://dx.doi.org/10.1111/j.1572-0241.2007.01562_9.x

[23] Sigal, S.H., Stanca, C.M., Kontorinis, N., Bodian, C. and Ryan, E. (2006) Diabetes Mellitus Is Associated with Hepatic Encephalopathy in Patients with HCV Cirrhosis. American Journal of Gastroenterology, 101, 1490-1496. http://dx.doi.org/10.1111/j.1572-0241.2006.00649.x 